\title{
Comparative chloroplast proteome analysis of exogenously supplied trehalose to wheat seedlings under heat stress
}

\author{
Y. LUO ${ }^{+}$, H.Y. LIU, Y.Z. FAN, W. WANG, and Y.Y. ZHAO \\ Instruments Sharing Platform of School of Life Sciences, East China Normal University, Shanghai 200241, China
}

\begin{abstract}
The aim of our study was to investigate the underlying molecular mechanisms of exogenously supplied trehalose affecting wheat photosynthesis under heat stress. The amount of ATP synthase (ATPase), oxygen-evolving enhancer protein (OEE), PsbP, Rubisco, chloroplast fructose-bisphosphate aldolase (FBPA), and ferredoxin-NADP(H) oxidoreductase (FNR) were downregulated, while PSI reaction center subunits were upregulated under heat stress. However, in the trehalose-pretreated groups, the amount of FNR, cytochrome $b_{6} f$ complex, PSI reaction center subunits, ATPase, FBPA, and Rubisco were upregulated under normal growth conditions and heat stress. Besides, during the recovery period, the upregulation in $\mathrm{CAB}$, PsbP, OEE2, and ATPase suggested that trehalose pretreatment might help to the recovery of PSII and PSI. These results indicate that trehalose pretreatment effectively regulates the levels of the photosynthesis-related proteins and relieves the damage of heat stress to wheat chloroplast.
\end{abstract}

Additional key words: chlorophyll fluorescence; chloroplast; photosynthesis-related proteome; PSI activity.

\section{Introduction}

The ambient temperature has increased steadily during this century (IPCC 2008). This continued warming trend may increase the frequency and severity of heat stress which can affect wheat photosynthesis, respiration, and uptake of mineral elements etc., thereby restricting the wheat growth and affecting the crop yield and quality of wheat. Wheat photosynthesis is very sensitive to high temperature (Wahid et al. 2007), which can reduce photosynthesis by destroying the structure and function of chloroplast and decreasing the chlorophyll (Chl) content (Qiang et al. 1995), and thus accelerate leaf senescence, and finally limit the growth and yield of wheat (Wang et al. 2011). Heat disasters often happen in the north of China and the Yangtze River region and reduce wheat production by 10 $20 \%$ (Jin 1983). Therefore increasing the heat tolerance of wheat is very important during the wheat cultivation in the present situation.
Trehalose, widely distributed in nature, is a nonreducing disaccharide composed of two glucose units linked at their 1-C (Richards et al. 2002). Due to the characteristics of its chemical composition, trehalose can protect cellular components, including proteins and cell membrane under stress conditions. Therefore, trehalose is a kind of stress protectant. Wheat (El-Bashiti et al. 2005, Luo et al. 2010), rice (Garg et al. 2002), and yeast (Baudoinia compniacensis) (Al-Naama et al. 2009) can accumulate trehalose to protect their cells from the further damages and maintain themselves under normal conditions. Genetic approaches show that the expression of trehalose-biosynthetic genes (TPS and/or TPP genes) from yeast and $E$. coli in tomato and some other model plants increases their abiotic stress tolerance and improves photosynthesis (Lyu et al. 2013). However, in most cases, trehalose accumulation is too low to serve as an

Received 31 January 2017, accepted 28 July 2017, published as online-first 12 April 2018.

${ }^{+}$Corresponding author, e-mail: 1081 yin@,163.com

Abbreviations: $\mathrm{CAB}$ - chlorophyll $a-b$ binding protein; $\mathrm{CEF}$ - cyclic electron flow; $\mathrm{Chl}$ - chlorophyll; Cyt $b_{6} f-$ cytochrome $b_{6} f$ complex; $\mathrm{ETR}_{(\mathrm{I})}$ - electron transfer rate of PSI; ETR $\left(\right.$ II) - electron transfer rate of PSII; $\mathrm{F}_{0}$ - minimal fluorescence yield of the dark-adapted state; $\mathrm{F}_{\mathrm{m}}$ - maximum fluorescence; $\mathrm{F}_{\mathrm{v}} / \mathrm{F}_{\mathrm{m}}$ - the maximum quantum yield of PSII photochemistry; FAD - flavin adenine dinucleotide; FBPA - chloroplast fructose-bisphosphate aldolase; FM - fresh mass; FNR - ferredoxin-NADP(H) oxidoreductase; IEF - isoelectric focusing; MS - mass spectrometry; OEC - oxygen-evolving complex; OEE - oxygen-evolving enhancer protein; $\mathrm{P}_{\mathrm{m}}$ - maximal P700 signal; PsaD - PSI reaction center subunit II; PsaE - PSI reaction center subunit IV; $\mathrm{qP}_{\mathrm{P}}$ - photochemical quenching coefficient; SD - standard deviation; TIL - temperature stress-induced lipocalin; $\mathrm{Y}_{(\mathrm{I})}$ - photochemical quantum yield of PSI; $\mathrm{Y}_{(\mathrm{II})}$ - effective photochemical quantum yield of PSII; $Y_{(\mathrm{NA})}$ - nonphotochemical PSI quantum yield of acceptor-side; $\mathrm{Y}_{(\mathrm{ND})}$ - nonphotochemical PSI quantum yield of donor-side; $\mathrm{Y}_{(\mathrm{NO})}$ - PSII quantum yield of nonregulated energy dissipation; $\mathrm{Y}_{(\mathrm{NPQ})}$ - PSII quantum yield of regulated energy dissipation. Acknowledgements: This work was financially supported by the Natural Science Foundation of China (No. 31200301). 
osmoprotectant molecule (Wingler and Paul 2013). For example, the trehalose content detected in rice and tobacco is only about $10 \mu \mathrm{g} \mathrm{g}^{-1}$ (fresh mass, FM) and it also varies in different species. In our previous study, the winter wheat synthesized $502 \mu \mathrm{g}$ (trehalose) $\mathrm{g}^{-1}(\mathrm{FM})$ under normal growth conditions, which increased to $1,250 \mu \mathrm{g} \mathrm{g}^{-1}(\mathrm{FM})$ under heat stress and to $1,658 \mu \mathrm{g} \mathrm{g} \mathrm{g}^{-1}(\mathrm{FM})$ in trehalosepretreated seedlings (Luo et al. 2010), indicating that exogenously supplied trehalose can effectively increase the trehalose content in wheat and act as a protectant.

Recently, with the development of proteomics, we can learn more about the complex molecular mechanisms of plant responses to heat stress at the protein level. Except wheat, many crops, such as rice, barley, and soybean, have been studied in proteomics. Chloroplast, the organelle of photosynthesis, also possesses genetic material. However, its genome only encodes about $80-100$ different kinds of proteins and the other about 2,500-3,500 different kinds of proteins are encoded by the nuclear genome (Martin and

\section{Materials and methods}

Plant growth and treatments: Seedlings of wheat (Triticum aestivum L. cv. Ning 13) grew hydroponically in Hoagland solution as described in Luo et al. (2008), in a 13-h day [PAR of $300 \mu \mathrm{mol}$ (photon) $\mathrm{m}^{-2} \mathrm{~s}^{-1}$ ] and 11-h night regime. When the second leaves were fully expanded, wheat seedlings were treated with Hoagland solution containing $1.5 \mathrm{mM}$ trehalose for $3 \mathrm{~d}$ (Tre) or with Hoagland solution alone as a control (CK). After that, the seedlings were subjected to heat stress $\left(40^{\circ} \mathrm{C}\right.$ for $\left.24 \mathrm{~h}\right)$, and then were kept at room temperature for another $12 \mathrm{~h}$ (for 2-D) and $24 \mathrm{~h}$ (for the determination of Chl fluorescence and P700) which were marked as R12 or R24, respectively.

Chl fluorescence and P700 measurements were carried out simultaneously using Dual-PAM-100 fluorometer (Walz, Effeltrich, Germany) and the parameters were assessed using the induction curve recording mode in the $D U A L-P A M$ software. After being for 30 min adapted in the dark, the minimal fluorescence yield $\left(\mathrm{F}_{0}\right)$ was immediately established. Then the maximum fluorescence $\left(\mathrm{F}_{\mathrm{m}}\right)$ was detected using saturation pulse $[10,000 \mu \mathrm{mol}$ (photon) $\mathrm{m}^{-2} \mathrm{~s}^{-1}, 600-\mathrm{ms}$ duration] and maximal quantum yield of PSII $\left(\mathrm{F}_{\mathrm{v}} / \mathrm{F}_{\mathrm{m}}\right)$ was calculated as $\left(\mathrm{F}_{\mathrm{m}}-\mathrm{F}_{0}\right) / \mathrm{F}_{\mathrm{m}}$ (Kramer et al. 2004; Schreiber 2004). A 620-nm lightemitting diode (LED) light and a PAR of 531 $\mu$ mol(photon) $\mathrm{m}^{-2} \mathrm{~s}^{-1}$ from 460-nm LED blue actinic light were delivered to the wheat seedlings. The effective photochemical quantum yield of PSII [ $\left.\mathrm{Y}_{(\mathrm{II})}\right]$ and photochemical quenching $\left(\mathrm{q}_{\mathrm{P}}\right)$ were automatically calculated by the $D U A L-P A M$ software. After far-red preillumination, the maximal $\mathrm{P} 700$ signal $\left(\mathrm{P}_{\mathrm{m}}\right)$ was detected by applying a single pulse. $\mathrm{P}_{\mathrm{m}}$ ' is analogous to the Chl fluorescence parameter $\mathrm{F}_{\mathrm{m}}$ '. The nonphotochemical PSI quantum yield of acceptor side-limited heat dissipation $\left[\mathrm{Y}_{(\mathrm{NA})}\right]$ was calculated as $\mathrm{Y}_{(\mathrm{NA})}=\left(\mathrm{P}_{\mathrm{m}}-\mathrm{P}_{\mathrm{m}}{ }^{\prime}\right) / \mathrm{P}_{\mathrm{m}}$ (Pfündel 2008). The
Herrmann 1998). Meanwhile, the functions of many proteins in chloroplast have not been thoroughly studied. Moreover, there is no detailed analysis about the influence of trehalose on wheat chloroplast proteome under heat stress. Our previous work showed that exogenously supplied trehalose protected chloroplast thylakoid membrane structure in a concentration-dependent manner (Luo et al. 2010). Therefore, we speculate that, under heat stress, exogenously supplied trehalose may also have influence on chloroplast proteome. In this paper, $1.5 \mathrm{mM}$ trehalose is exogenously supplied to wheat seedlings and their physiologically relevant conditions are mimicked. We aimed to apply 2-DE technique to detect changes in the level of protein abundance and then identify the main proteins differentially expressed under heat stress. Meanwhile, we were looking forward to investigate the underlying molecular mechanism in response to exogenously supplied trehalose under heat stress in the wheat chloroplast.

donor side-limited heat dissipation $\left[\mathrm{Y}_{(\mathrm{ND})}\right]$, the photochemical quantum yield of PSI $\left[\mathrm{Y}_{(\mathrm{I})}\right]$ and the electron transfer rate of PSI $\left[\mathrm{ETR}_{(\mathrm{I})}\right]$ were also automatically calculated by the software. All the measurements were repeated for at least 5 times.

Isolation and purification of chloroplasts: The collected leaves of wheat seedlings (the second and third leaves) were used to extract chloroplasts. The isolation protocols of chloroplasts from wheat leaves were performed according to Wang et al. (2013) with some modifications. All steps were performed in the dark at $4^{\circ} \mathrm{C}$. Frozen wheat leaves were cut into small pieces with scissors and grounded to fine powder with mortar and pestle in liquid nitrogen. Then, isolation buffer I, containing $0.33 \mathrm{M}$ sorbitol, $1 \mathrm{mM} \mathrm{MgCl}_{2}, 50 \mathrm{mM} \mathrm{HEPES} / \mathrm{KOH}$ (pH 7.8), $2 \mathrm{mM}$ EDTA, $10 \mathrm{mM}$ DTT, $0.1 \%$ BSA, $5 \mathrm{mM}$ sodium ascorbate, and $0.1 \%$ polyvinylpyrrolidone (PVP), was added. The homogenate was filtered through four layers of gauze. Then the filtrate was centrifuged at $2,000 \times g$ for 10 min to harvest the crude chloroplasts. Next, the pellets were carefully resuspended in $5 \mathrm{~mL}$ of the isolation buffer II, containing $0.3 \mathrm{M}$ sorbitol, $1 \mathrm{mM} \mathrm{MgCl}_{2}, 50 \mathrm{mM}$ HEPES/KOH (pH 7.8), and 2 mM EDTA. Subsequently, the resuspended chloroplasts were loaded onto a Percoll step gradient buffer, which was combined with $5 \mathrm{~mL}$ of $40 \%$ Percoll and $4 \mathrm{~mL}$ of $80 \%$ Percoll in the isolation buffer II. Chloroplast separation was achieved by centrifugation at $16,000 \times g$ for $20 \mathrm{~min}$ at $4^{\circ} \mathrm{C}$. The green interphase between 40 and $80 \%$ Percoll was the purified intact chloroplasts. Then, the isolation buffer II was added to the chloroplasts and washed twice. The pellets were used immediately following the protein extraction protocols or stored at $-80^{\circ} \mathrm{C}$. 
Extraction of chloroplast proteins was carried out as described by Phee et al. (2004) with some modifications. The chloroplast preparations were lysed in $1 \mathrm{~mL}$ of solubilization buffer (50 mM HEPES/KOH, pH 7.9, 4\% CHAPS, $1 \mathrm{mM}$ PMSF) and incubated on ice for at least $2 \mathrm{~h}$ and then centrifuged at $12,000 \times g$ for $15 \mathrm{~min}$ at $4^{\circ} \mathrm{C}$. Four volumes of $100 \%$ acetone were added into the supernatant, and it was stored at $-20^{\circ} \mathrm{C}$ to $-25^{\circ} \mathrm{C}$ for at least $2 \mathrm{~h}$. Chloroplast proteins were collected by centrifugation at $15,000 \times g$ for $15 \mathrm{~min}$. Pellets were washed with precold acetone $\left(-20^{\circ} \mathrm{C} \sim-25^{\circ} \mathrm{C}\right)$ for 3 times. Finally, the washed pellets were air-dried and ready for use or stored at $-80^{\circ} \mathrm{C}$.

2-D gel electrophoresis and isoelectric focusing: All the 2-D procedures were performed according to the manual of Bio-Rad. Protein samples were resuspended in a lysis buffer (7 M urea, $2 \mathrm{M}$ thiourea, 4\% CHAPS, $40 \mathrm{mM}$ DTT, $0.2 \%$ Bio-Lyte) at room temperature for $30 \mathrm{~min}$ in order to achieve full denaturation, and then centrifugated at $20,000 \times g$ for $15 \mathrm{~min}$ at room temperature. The supernatant contained the fully denatured proteins. Protein quantification was carried out using the Bradford protein assay kit. After quantification, a trace of bromophenol blue was added and mixed. For isoelectric focusing (IEF), immobilized gel strips ( $\mathrm{pH} 4-7 \mathrm{~L}, 17 \mathrm{~cm}$; Bio-Rad, USA) were used to separate the protein lysate (the IEF temperature was set at $20^{\circ} \mathrm{C}$ ). The procedure was modified for full and steady-state focusing. Briefly, the gel strip was rehydrated for $12 \mathrm{~h}$ at $50 \mathrm{~V}$ and the proteins were separated using the following stepwise increases in voltage and running times: $250 \mathrm{~V}$ for $30 \mathrm{~min} ; 1,000 \mathrm{~V}$ for $1 \mathrm{~h} ; 10,000$ $\mathrm{V}$ for $5 \mathrm{~h}$; and then $10,000 \mathrm{~V}$ ( to a total for $60 \mathrm{kVh}$ ). After IEF, strip gels were incubated for $15 \mathrm{~min}$ at room temperature with equilibration buffer I $(0.375 \mathrm{M}$ Tris- $\mathrm{HCl}$, $\mathrm{pH} 8.8,6 \mathrm{M}$ urea, 20\% glycerol, 2\% SDS, 1\% DTT) and then transferred to equilibration buffer II $(0.375 \mathrm{M}$ Tris$\mathrm{HCl}, \mathrm{pH} 8.8,6 \mathrm{M}$ urea, 30\% glycerol, 2\% SDS, 2.5\% iodoacetamide) for another $15 \mathrm{~min}$. After this equilibration step, the strip gels were placed on $12.5 \%$ denaturing

\section{Results}

Chl fluorescence and P700 measurement: Chl fluorescence is often used to measure the changes of PSII activity under stress conditions. In the $\mathrm{CK}$ groups, $\mathrm{F}_{\mathrm{v}} / \mathrm{F}_{\mathrm{m}}$ declined under heat stress (Fig. 1A). Meanwhile, a similar pattern of other parameters including $\mathrm{Y}_{(\mathrm{II})}($ Fig. $1 B)$, $\mathrm{q}_{\mathrm{P}}($ Fig. $1 C$ ), and electron transfer rate in PSII [ETR $($ II) (Fig. 1D) was observed under heat stress. However, in the Tre groups, these parameters were significantly higher than those in the CK groups under heat stress and during heat recovery period, and maintained at the similar levels to the nonstressed seedlings (Fig. 1). These results indicated that exogenously supplied trehalose could relief the damage of heat stress to the photosynthetic apparatus, and thus diminish its adverse effect on the photochemical efficiency. acrylamide gels and sealed with a $0.5 \%$ agarose solution. SDS-PAGE was performed at $150 \mathrm{~V}$ for $8 \mathrm{~h}$ with a PROTEAN II xl 2-D Cell (Bio-Rad, USA). Protein spots in the 2-D gels were visualized by Coomassie Brilliant Blue (CBB G-250) staining.

Image analysis and protein identification: To analyze the expressed protein patterns, stained gels were scanned using a Powerlook III flatbed scanner (UMAX, Taiwan), followed by analysis of protein spots using PDQuest $v 8.0$ (Bio-Rad, USA). The differentially expressed proteins were chosen by the spot density between different groups. The target protein spots were excised from the stained gels and transferred to Sangon Corporation (Shanghai, China) for Maldi-TOF/TOF-MS (Applied Biosystems, Foster City, USA). In gel digestion was performed according to Katayama et al. (2001). Samples were resuspended with $5 \mu \mathrm{L}$ of $0.1 \%$ TFA followed by mixing in $1: 1$ ratio with a matrix consisting of a saturated solution of $\alpha$-cyano-4hydroxy-trans-cinnamic acid in 50\% acetonitrile (ACN), $0.1 \%$ TFA, and then peptide mass spectrometry (MS) and MS/MS analyses were performed. Based on combined MS and MS/MS spectra, proteins were successfully identified according to $95 \%$ or higher confidence interval of their scores in the MASCOT V2.3 search engine (Matrix Science Ltd., London, U.K.) against the NCBInr-Viridiplantae (green plants) database. The digestion enzyme was trypsin, and carbamidomethyl was considered as the fixed modification, while acetyl (protein N-term), deamidated, dioxidation, and oxidation were considered as partial modifications. One single missed cleavage, $100 \mathrm{ppm}$ for precursor ion tolerance and $0.3 \mathrm{Da}$ for fragment ion tolerance was permitted.

Statistical analysis: All comparisons were analyzed by one-way analysis of variance (ANOVA), LSD and Tukey's test of multiple comparison analysis for the independent samples $(p<0.05)$. Values were expressed as mean $\pm \mathrm{SE}$ from at least three independent experiments.

Meanwhile, heat stress induced the increase in $\mathrm{Y}_{(\mathrm{NO})}$ and the decrease in $\mathrm{Y}_{(\mathrm{NPQ})}$ (Fig. $\left.1 E, F\right)$, and the $\mathrm{Y}_{(\mathrm{NO})}$ in the Tre group was lower than that in CK group (Fig. 1E).

P700 parameters reflect a state of PSI. Heat stress decreased the photochemical quantum yield of PSI [Y $\left.\mathrm{Y}_{(\mathrm{I})}\right]$ (Fig. 2D) and $\mathrm{ETR}_{(\mathrm{I})}$ (Fig. 2E) compared with CK groups. But the maximal $\mathrm{P} 700$ signal $\left(\mathrm{P}_{\mathrm{m}}\right)$ (Fig. $\left.2 A\right)$ showed no significant changes during heat stress, which indicated that the photosynthetic activity of PSI may be generally stable under heat stress and during the subsequent recovery period. Meanwhile, Tre pretreatment made no significant effects on P700 parameters (Fig. 2) under normal condition, but $\mathrm{Y}_{(\mathrm{ND})}($ Fig. $2 B$ ) significantly increased under heat stress, indicating that the light protection mechanism for PSI in 


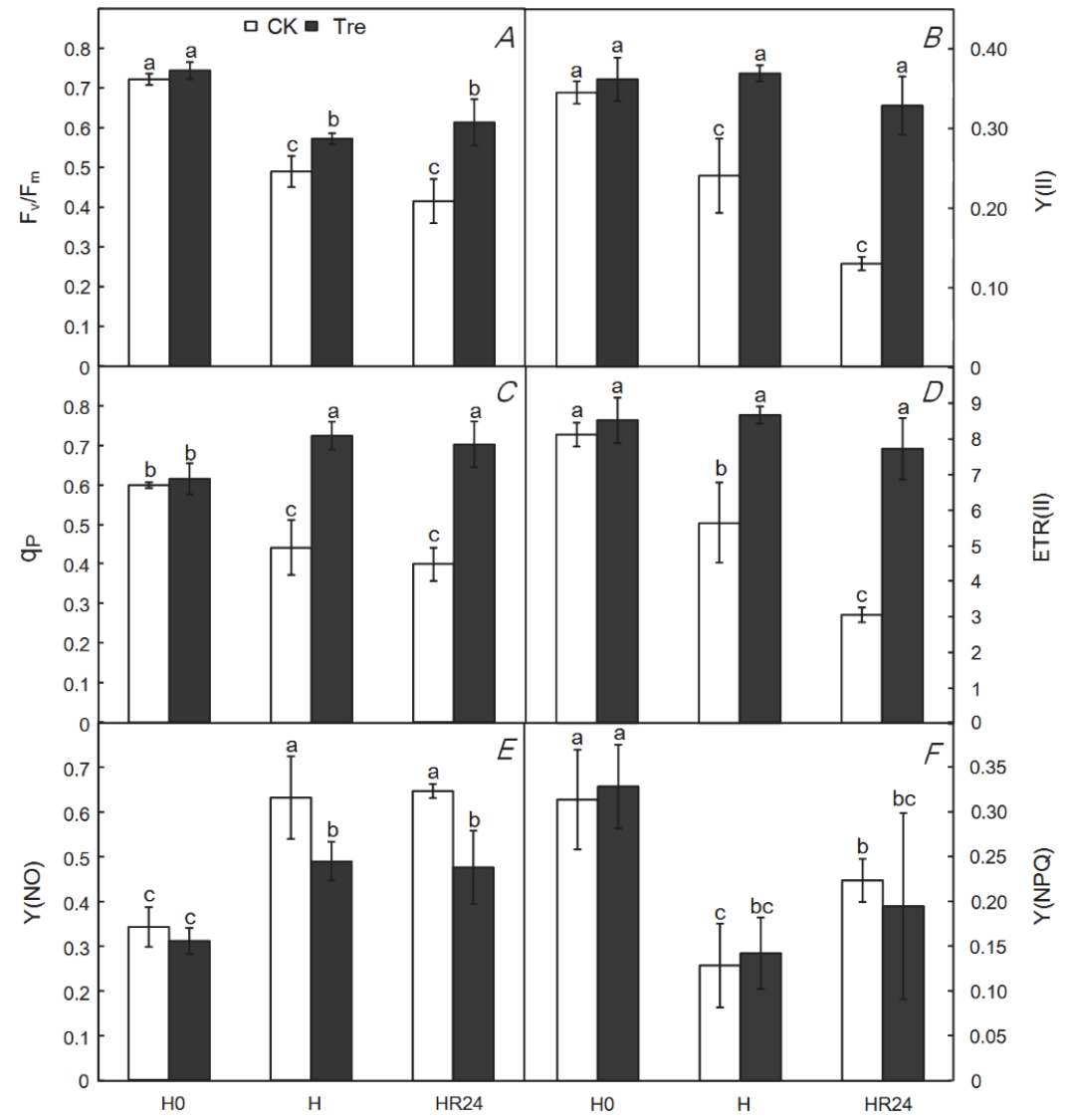

Fig. 1. Influence of exogenous trehalose on chlorophyll fluorescence parameters: $\mathrm{F}_{\mathrm{v}} / \mathrm{F}_{\mathrm{m}}$ $(A), \mathrm{Y}(\mathrm{II})(B), \mathrm{qP}(C), \mathrm{ETR}(\mathrm{II})(D), \mathrm{Y}(\mathrm{NO})$ $(E)$, and $\mathrm{Y}(\mathrm{NPQ})(F)$ of wheat leaves under different treatments. CK - control group; Tre - trehalose pretreatment for $3 \mathrm{~d}$; H0 - without high temperature treatment; $\mathrm{H}$ - high temperature treatment for $24 \mathrm{~h}$; HR24 - recovered $24 \mathrm{~h}$ from high temperature treatment. Different letters show significant differences $(P<0.05)$.
$\mathrm{Y}_{(\mathrm{ND})}$ enhanced (Fig. 2B), but the $\mathrm{Y}_{(\mathrm{I})}$ (Fig. 2D) was lowered at the same time, indicating that the light protection mechanism for PSI was promoted to a certain extent.

Wheat chloroplast proteome: Through image analysis with PDQuest software, more than 100 highly reproducible chloroplast protein spots were identified on each gel (Fig. 3). From these spots based on three replications, 19 spots were revealed as differentially expressed proteins with pI values ranging from 4 to 7 and with relative molecular masses of 10 to $200 \mathrm{kDa}$.

Compared with CK plants, Tre pretreatment upregulated the amount of cytochrome $b_{6} f$ complex (Cyt $b_{6} f$ ) iron-sulfur subunit, ferredoxin-NADP $(\mathrm{H})$ oxidoreductase (FNR), and PSI reaction center subunit II (PsaD), ATP synthase (ATPase) subunit gamma in the photosynthetic electron transport chain. Besides, the chloroplast fructosebisphosphate aldolase (FBPA), Rubisco large subunit in the carbon reaction were also upregulated (Fig. 3A). However, the contents of PSI reaction center subunit IV (PsaE), PsbP, OEE2, and the predicted chloroplastic-like protein TIC110 were downregulated (Fig. $3 A$, Table 1).

Under heat stress, Tre pretreatment significantly increased the amount of ATPase CF1 beta subunit and FBPA (Fig. 3B, Table 1). Meanwhile, the contents of FNR were also upregulated (Fig. $3 B$, Table 1). In contrast, the amount of the proteins including Cyt $b_{6} f$ iron-sulfur subunit 2, Chl $a-b$ binding protein (CAB), Rubisco large subunit, chloroplastic ATPase delta chain, PsbP, OEE2, and the hypothetical protein TRIUR3 05353 were downregulated in wheat (Fig. $3 B$, Table 1).

After recovery from heat stress, compared with the CK (without Tre pretreatment), Tre pretreatment upregulated the levels of CAB, FBPA, PsbP, OEE2, ATPase delta chain, and CF1 alpha subunit, while the amount of ATPase CF1 beta subunit, OEE1, temperature stress-induced lipocalin (TIL), Rubisco large subunit, and FNR were generally downregulated (Fig. $3 C$, Table 1). Besides, some hypothetical proteins, such as hypothetical protein TRIUR3_26766, the predicted chloroplastic-like protein TIC110, and plastid-lipid-associated protein 13, also showed lower contents in Tre groups than those in CK plants (Fig. 3C, Table 1).

Under heat stress, the amount of Cyt $b_{6} f$ iron-sulfur subunit 2, CAB, PsaD, PsaE, the predicted chloroplasticlike protein TIC110, and plastid-lipid-associated protein 13 in wheat chloroplasts were upregulated (Fig. 3D, Table 1). However, the levels of OEE1 and OEE2, ATPase delta chain, ATPase CF1 alpha subunit, ATPase beta subunit, Rubisco large subunit, FBPA, FNR, and PsbP showed the downregulation in the heat-stressed groups (Fig. 3D, Table 1). 


\section{Discussion}

Previously, we found that the proteins in the thylakoid membranes and photosynthetic capacity damaged by heat stress could be protected by Tre pretreatment in wheat seedlings (Luo et al. 2010). However, in this study, when exposed to heat stress, the level of CAB, which serves to maximize and regulate light harvesting (Silva et al. 2016), was upregulated (Fig. 3D, 3.19 \pm 0.19 ). Silva et al. (2016) reported that the $P g C A B 1$ and $P g C A B 2$ expression levels significantly increased compared with control under $\mathrm{NaCl}$ stress, which suggested the specific function of PSII in the regulatory role in ginseng response to salinity and drought and this also might represent a strategy to prevent light stress-induced damage. We speculated that the plant itself might depress its energy supply to photosynthesis because of the heat stress and thus hope to meet the needs of energy and its metabolic balance by enhancing light harvesting in order to respond to this decrease in photosynthesis. Similarly, the lower content of CAB in the Tre-pretreated groups compared with $\mathrm{CK}$ group under heat stress (Fig. 3B, $0.46 \pm 0.16$ ) may be due to the reason that other response ways have compensated some of the needs. In accordance with the enhancement of Chl fluorescence parameters including the $\mathrm{F}_{\mathrm{V}} / \mathrm{F}_{\mathrm{m}}, \mathrm{Y}_{(\mathrm{II})}$, and $\mathrm{q}_{\mathrm{P}}($ Fig. 1) in the
Tre groups under heat stress, the level of CAB 8 in Tre groups was also generally upregulated (Fig. 3C, $2.01 \pm$ 0.04 ) after recovery from heat stress, suggesting that during the subsequent recovery period, trehalose can promote plant metabolism by enhancing light harvesting, and therefore repair the damage caused by heat stress. The thermolability of PSII can depress photosynthetic electron transport in plants and may induce the ROS generation. And in the present work, we found that the ETR $_{(I I)}$ significantly decreased under heat stress (Fig. 1D). When exposed to heat stress, oxygen-evolving complex (OEC) would separate from PSII ( $\mathrm{Hu}$ et al. 2004) which is consistent with the downregulation of PsbP and OEE under heat stress (Fig. $3 D, 0.62 \pm 0.05 ; 0.62 \pm 0.05$ ). It was reported that PsbP, one of the extrinsic OEC proteins, is essential for the normal PSII function in higher plants in vivo (Ifuku et al. 2005), and it plays an important role in the dynamic life cycle of PSII (Ifuku et al. 2008, Ifuku et al. 2011). In this way, the upregulation of the PsbP content in the Tre-treated group during the recovery period helps to the recovery of PSII, which is in agreement with the result reported by Mamedov et al. (2015) that trehalose significantly stimulates the steady-state rate of $\mathrm{O}_{2}$

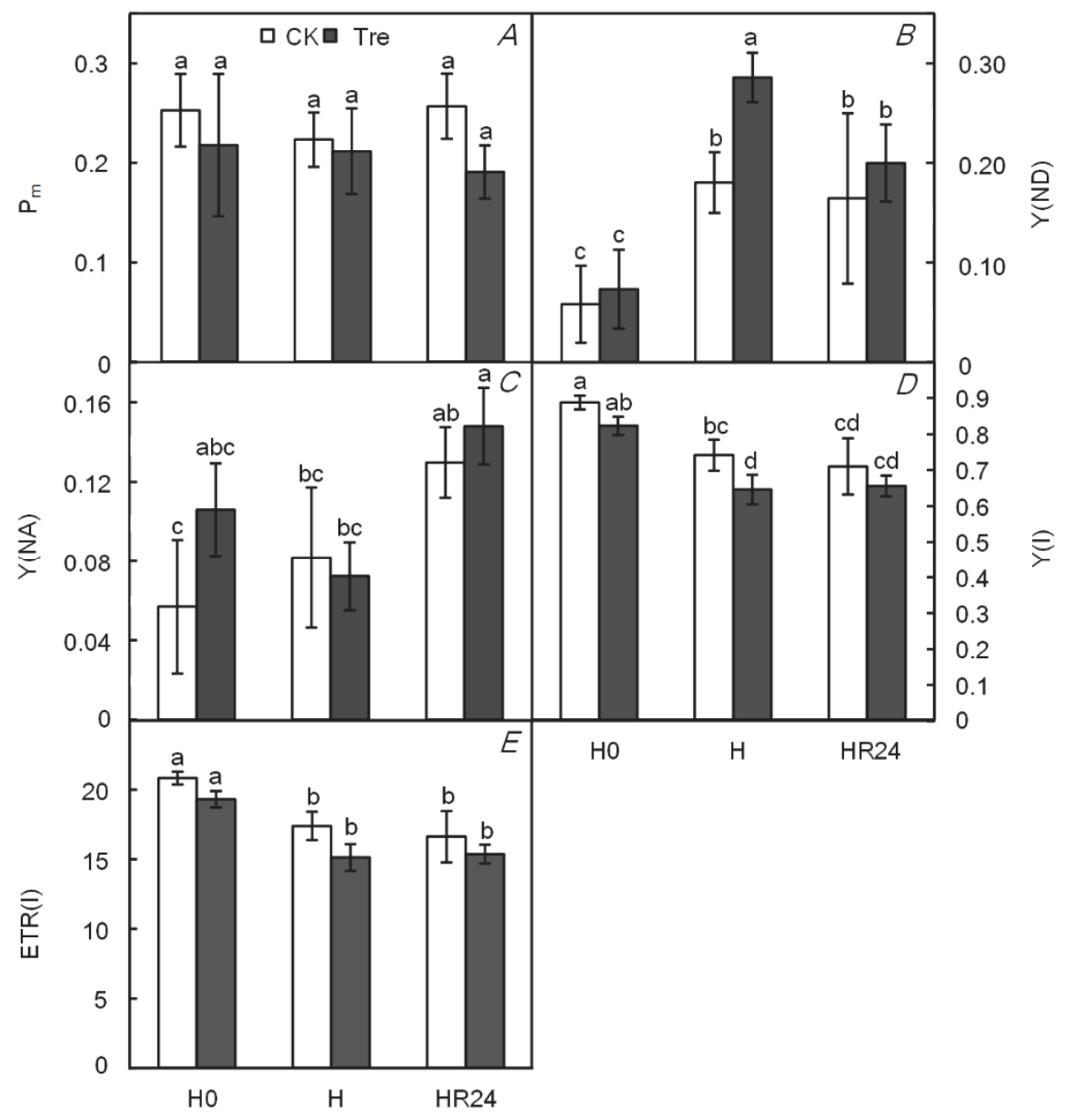

Fig. 2. Influence of exogenous trehalose on P700 parameters: $\mathrm{P}_{\mathrm{m}}(A), \mathrm{Y}(\mathrm{ND})(B)$, $\mathrm{Y}(\mathrm{NA})(C), \mathrm{Y}(\mathrm{I})(D)$ and $\operatorname{ETR}(\mathrm{I})(E)$ of wheat leaves under different treatments. $\mathrm{CK}$ - control group; Tre - trehalose pretreatment for $3 \mathrm{~d}$; $\mathrm{H} 0$ - without high temperature treatment; $\mathrm{H}$ - high temperature treatment for $24 \mathrm{~h}$; HR24 recovered $24 \mathrm{~h}$ from high temperature treatment. Different letters show significant differences $(P<0.05)$. 


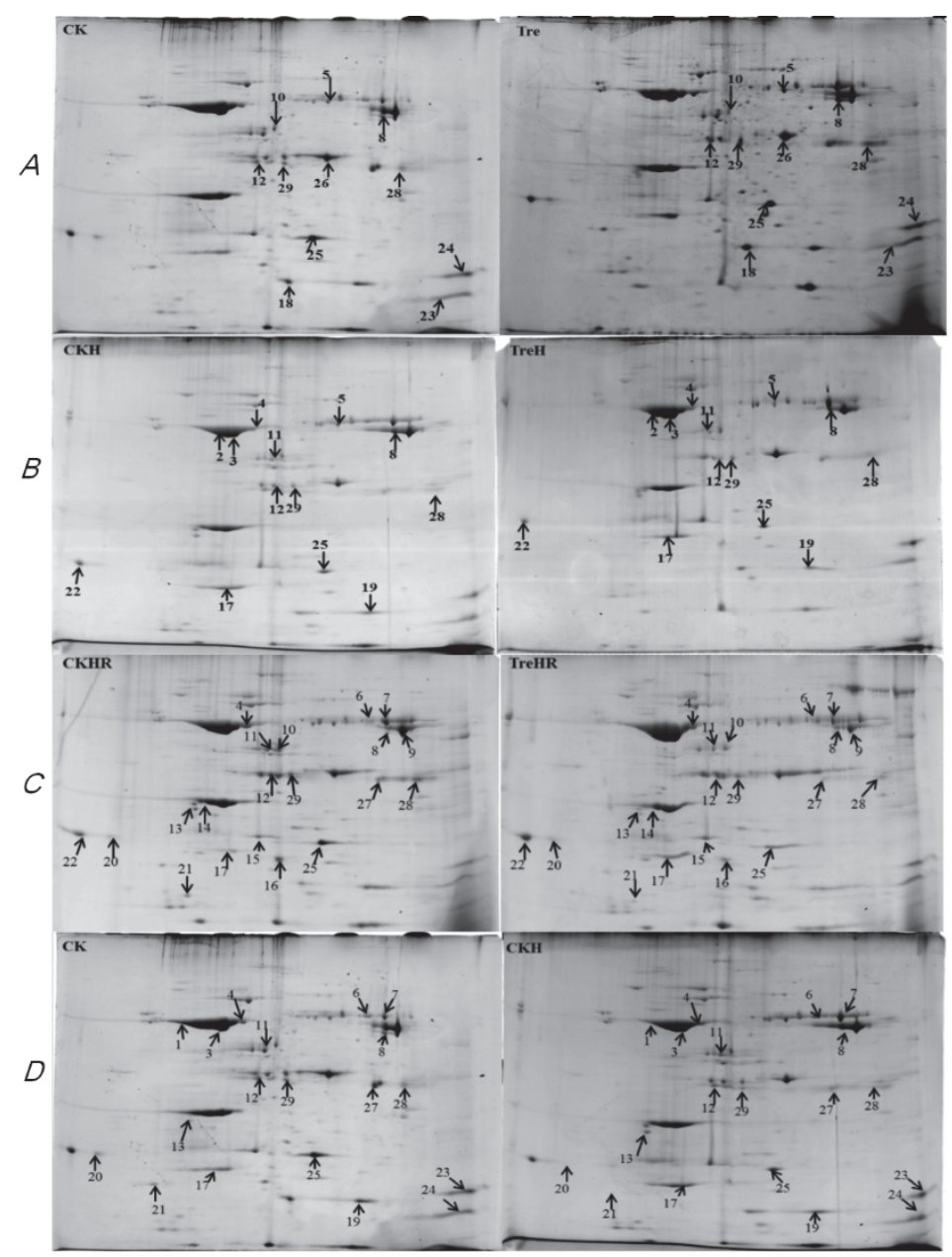

Fig. 3. Effects of exogenously supplied trehalose and heat stress on protein levels in wheat control seedlings $(\mathrm{CK})$ and plants that pretreated with trehalose for $3 \mathrm{~d}$ (Tre). (A) gel from the plants without heat stress, $(B)$ gel from the plants suffered from heat stress for $24 \mathrm{~h},(C)$ gel from the plants recovered for $12 \mathrm{~h},(D)$ gel from the plants before heat stress and after heat stress. $\mathrm{H}$ - high temperature treatment for $24 \mathrm{~h}$; HR - recovered from high temperature treatment. Proteins were extracted from leaf chloroplasts, separated by $2-\mathrm{DE}$, and visualized by CBB G-250 staining. The numbers were the ID of these proteins. evolution in both PSII membrane fragments and core complexes from spinach.

PsaD and PsaE are two subunits in PSI reaction center; PsaE is associated with cyclic electron flow (CEF) around PSI. They both were upregulated in wheat seedlings in the $\mathrm{CK}$ and Tre groups under heat stress (Fig. $3 B, D, 3.15 \pm$ $0.68,2.46 \pm 0.36)$. The upregulated PsaE under heat stress was in agreement with the result that heat stress significantly increased $\mathrm{Y}_{(\mathrm{ND})}$ (Fig. $2 B$ ), suggesting that the light protection mechanisms of PSI in wheat seedlings improved under heat stress. Morgan-Kiss et al. (2002) suggested that suitable high temperature could improve the electron transfer ability mediated by PSI. And $\mathrm{Hu}$ et al. (2004) reported that the heat stress had no effect on the secondary structure of PSI when the temperature is below $50^{\circ} \mathrm{C}$. PSI-CEF was confirmed to protect PSI against photodamage in heat-stressed leaves. So, the stable PSI activity may be due to the upregulated PSI subunits under heat stress. Our results were consistent with previous study by Zhang and Liu (2016) that heat stress $\left(25-40^{\circ} \mathrm{C}\right)$ increased the PSI activity in Arthrospira (Spirulina).

It was reported that when exposed to $42^{\circ} \mathrm{C}$ for $12 \mathrm{~h}$, the content of FNR in soybean leaves was diminished (Ahsan et al. 2010). Heat stress-induced downregulation in the protein level of FNR (Fig. 3D, 0.42 $\pm 0.10-0.55 \pm 0.09$ ) slowed down the process of photosynthetic electron transport, while under the Tre-pretreatment conditions, the upregulation of FNR (Fig. 3A,B, $1.73 \pm 0.04,1.96 \pm 0.17$ ) helped protect wheat seedlings against the heat stress effects on the photosynthetic electron transfer process. Meanwhile, the amount of Cyt $b_{6} f$ iron-sulfur subunit also increased in the Tre-pretreated groups (Fig. 3A, $1.59 \pm$ $0.04)$. Previous studies showed that the formation of FNRCyt $b_{6} f$ contributed to the CEF process (Zhang et al. 2001; Szymańska et al. 2011) and we also found that exogenously supplied Tre stimulated CEF (data not published). Therefore, it is possible that Tre pretreatment may contribute to heat tolerance in wheat seedlings through the CEF-induced protection mechanism.

The downregulation in the protein levels of ATPase CF1 $\alpha$ (Fig. $3 D, 0.13 \pm 0.01$ ) and $\beta$ subunit (Fig. $3 D, 0.41$ \pm 0.14 ) and $\delta$ chain (Fig. $3 D, 0$ ) under heat stress indicated that heat stress can damage the energy metabolism process of wheat seedlings since the catalytic site for ATP synthesis is localized primarily on the $\beta$ subunit. Similar to the results reported by Laino et al. (2010) in durum wheat seeds, our results showed that the amount of ATP-synthase decreased under heat stress. Moreover, Tre pretreatment 


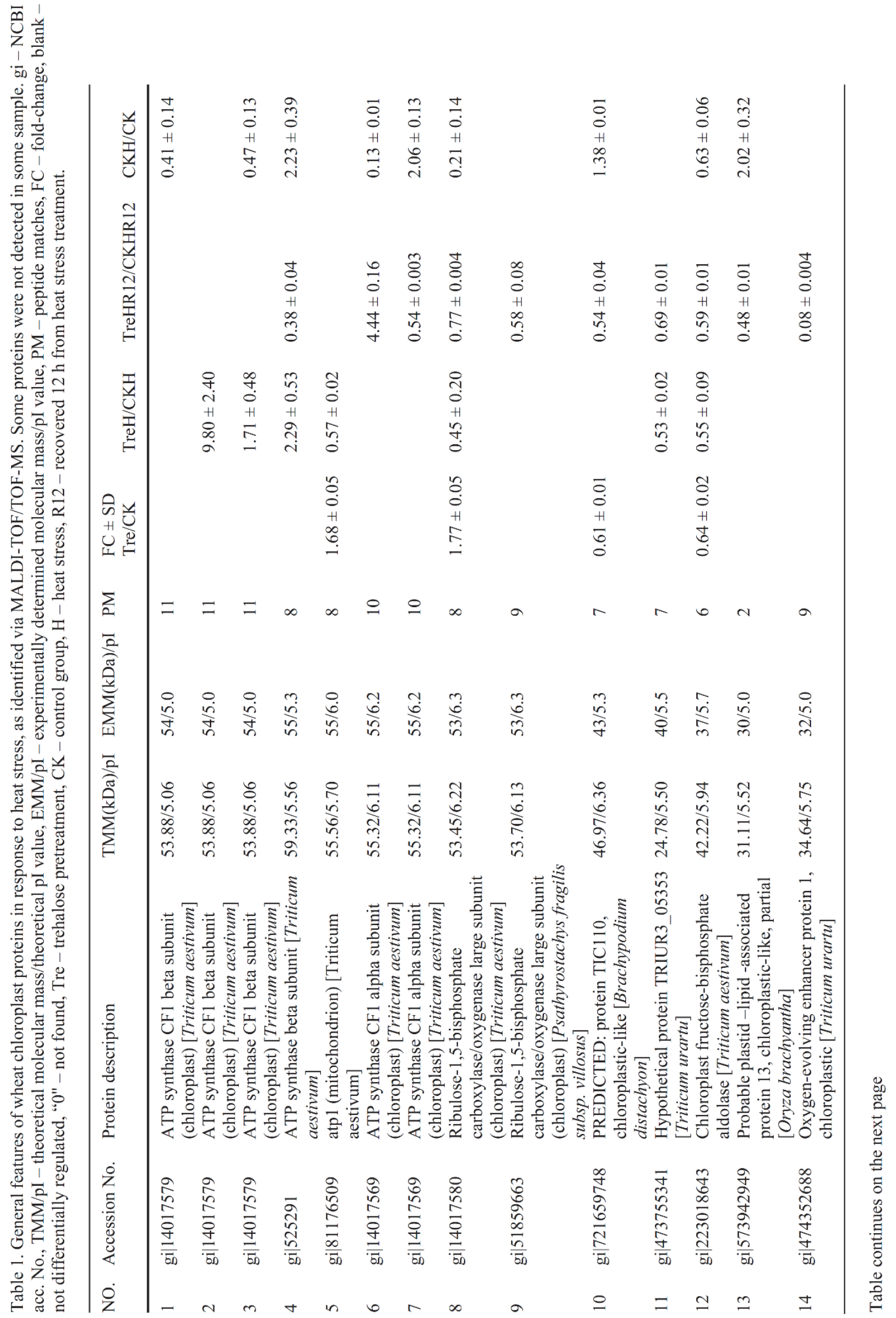




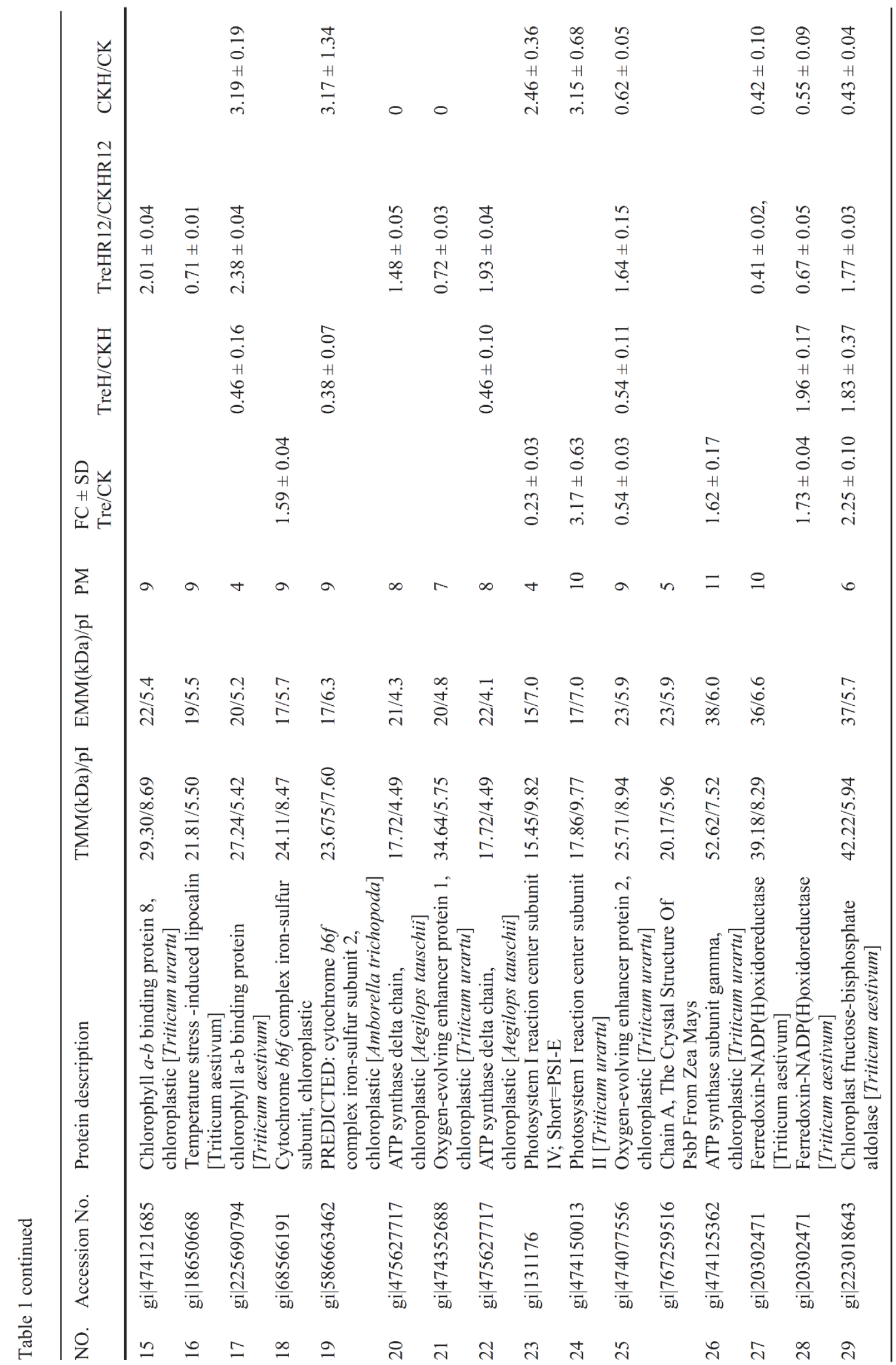




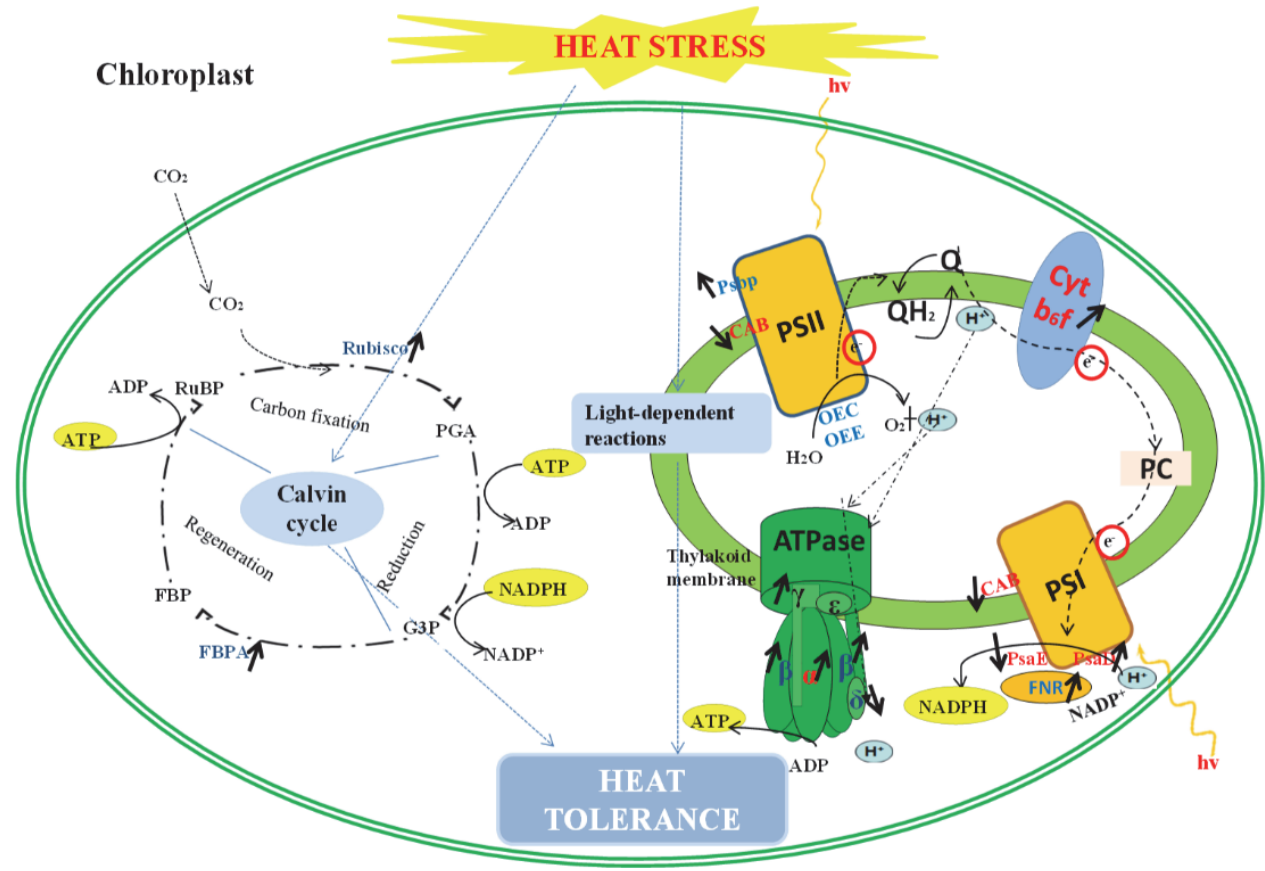

Fig. 4. Schematic presentation of a mechanism for heat tolerance in trehalose-pretreated wheat seedlings. This picture was redrawn from Wang et al. (2013) with some modification. Proteins under heat stress alone are integrated and indicated in red (upregulated) or blue (downregulated), respectively, while the protein level changes in trehalose pretreatment are indicated with arrows (up or down). ADP - adenosine diphosphate; ATP - adenosine triphosphate; ATPase - ATP synthase; CAB - chlorophyll $a / b$ binding protein; Cyt $b_{6} f$ cytochrome $b_{6} f$; FBP - fructose-1,6-bisphosphate; FBPA - fructose-bisphosphate aldolase; FNR - ferredoxin-NADP(H) oxidoreductase; G3P - glyceraldehydes-3-phosphate; LHC - light-harvesting complex; NADP+/NADPH - nicotinamide adenine dinucleotide phosphate; OEC - oxygen-evolving complex; OEE - oxygen-evolving enhancer protein; PC - plastocyanin; PGA - 3phosphoglycerate; Q - quinone; $\mathrm{QH}_{2}$ - reduced quinone; $\mathrm{RCP}$ - reaction center protein; Rubisco - ribulose-1,5-bisphosphate carboxylase/oxygenase; RuBP - ribulose-1,5-bisphosphate.

upregulated the amount of the ATPase $\gamma$ subunit (Fig. 3A, $1.62 \pm 0.17)$ before heat stress, while the amount of ATPase CF1 $\beta$ subunit was upregulated in Tre-pretreated groups during heat stress (Fig. 3B, $9.80 \pm 2.40$ ), suggesting that Tre can reduce the heat stress-induced damage to energy metabolism. It had also been proposed that the CF1 $\alpha$ subunit displays an organizing function during the assembly of ATP synthase when exposed to the thermal denaturation (Wang et al. 1993). Therefore, the upregulation of ATPase CF $1 \alpha$ subunit (Fig. 3C, $4.44 \pm 0.16$ ) in Tre-treatment group after recovery from heat stress possibly helped to re-establish the energy production system.

It is now well confirmed that increasing temperature inhibits photosynthetic $\mathrm{CO}_{2}$ fixation in many plant species largely by the decrease in the activation state of Rubisco (Majoul et al. 2004). Therefore, the upregulation in the protein content of Rubisco large subunit caused by exogenously supplied Tre (Fig. $3 A, 1.77 \pm 0.05$ ) might indicate that Tre pretreatment before heat stress helped strengthen photosynthesis efficiency. Moreover, the Tre pretreatment significantly enhanced the amount of FBPA in the chloroplast, and this would lead to the effective improvement in carbon assimilation process and therefore alleviated the inhibition by heat stress in plants.
The changes of proteins shown in Fig. 4 indicate that light-dependent reactions in Tre-pretreated seedlings were maintained by upregulating the component proteins including PsbP, Cyt $b_{6} f$, FNR, PsaD, and ATPase, while the normal function of the Calvin cycle was kept mainly by the upregulation of Rubisco and FBPA under heat stress. The upregulation of Cyt $b_{6} f$ helped enhance the CEF around PSI which is involved in the maintenance of ATP production. Meanwhile, the upregulation of ATPase and FNR in Tre-pretreated group also helped maintain the production of ATP and NADPH under heat stress which can lead to the reduced generation of ROS. Furthermore, the contents of other proteins were also changed, for example, TIL and plastid-lipid-associated protein 13 (Table 1). This might be due to the damage to chloroplast membrane system caused by heat stress. It is reported that the basal level of TIL under normal conditions is relatively high (Chi et al. 2009), and we only found that the TIL was upregulated visually during the recovery period compared to the normal and heat-stress conditions. This result was consistent with the MDA content under heat stress and the recovery period in our previous work (the MDA content increased after heat stress in both CK and Tre groups, but Tre-pretreatment alleviated the MDA increase during the recovery period) since it was proposed that TIL could 
protect plant against lipid peroxidation (Chi et al. 2009). The role of these proteins in heat tolerance is not known clearly. They may be directly related to heat stress response by an unknown mechanism or, indirectly, be a target for other proteins involved in the heat shock response (Laino et al. 2010). However, we did not detect the heat-shock protein in this experiment.

Conclusion: To the best of our knowledge, this current report is the first one to present changes in the chloroplast proteome of wheat seedlings pretreated with trehalose under heat stress. In conclusion, trehalose pretreatment

\section{References}

Ahsan N., Donnart T., Nouri M.Z. et al.: Tissue-specific defense and thermo-adaptive mechanisms of soybean seedlings under heat stress revealed by proteomic approach. - J. Proteome Res. 9: 4189-4204, 2010.

Al-Naama M., Ewaze J.O., Green B.J. et al.: Trehalose accumulation in Baudoinia compniacensis following abiotic stress. Int. Biodeter. Biodegr. 63: 765-768, 2009.

Chi W.T., Fung R.W.M., Liu H.C. et al.: Temperature-induced lipocalin is required for basal and acquired thermotolerance in Arabidopsis. - Plant Cell Environ. 32: 917-927, 2009.

El-Bashiti T., Hamamci H., Öktem H.A. et al.: Biochemical analysis of trehalose and its metabolizing enzymes in wheat under abiotic stress conditions. - Plant Sci. 169: 47-54, 2005.

Garg A.K., Kim J.K, Owens T.G. et al.: Trehalose accumulation in rice plants confers high tolerance levels to different abiotic stresses. - P. Natl. Acad. Sci. USA 99: 15898-15903, 2002.

Hu Z.H., Xu Y.N., Jiang G.Z. et al.: Degradation and inactivation of photosystem I complexes during linear heating. - Plant Sci. 166: 1177-1183, 2004

Ifuku K., Ido K., Sato F.: Molecular functions of PsbP and PsbQ proteins in the photosystem II supercomplex. - J. Photoch. Photobio. B 104: 158-164, 2011.

Ifuku K., Ishihara S., Shimamoto R. et al.: Structure, function, and evolution of the PsbP protein family in higher plants. Photosynth. Res. 98: 427-437, 2008.

Ifuku K., Yamamoto Y., Ono T.A. et al.: PsbP protein, but not PsbQ protein, is essential for the regulation and stabilization of photosystem II in higher plants. - Plant Physiol. 139: 11751184, 2005.

IPCC (Intergovernmental Panel on Climate Change): Intergovernmental Panel on Climate Change Fourth Assessment Report: Climate Change 2007. - Synthesis Report. Pp. 30-31. World Meteorological Organization, Geneva 2008.

Jin S.B.: Wheat cultivaters and their genealogy in China. - In: Jin S.B. (ed.): Wheat Cultivaters and their Genealogy in China ( $1^{\text {st }}$ ed.). Pp. 112-115. China Agriculture Press, Beijing 1983.

Katayama H., Nagasu T., Oda Y.: Improvement of in-gel digestion protocol for peptide mass fingerprinting by matrixassisted laser desorption/ionization time-of-flight mass spectrometry. - Rapid Commun. Mass Sp. 15: 1416-1421, 2001.

Kramer D.M., Johnson G., Kiirats O. et al.: New fluorescence parameters for the determination of $\mathrm{Q}_{\mathrm{A}}$ redox state and excitation energy fluxes. - Photosynth. Res. 79: 209-218, 2004.

Laino P., Shelton D., Finnie C. et al.: Comparative proteome analysis of metabolic proteins from seeds of durum wheat (cv. Svevo) subjected to heat stress. - Proteomics 10: 2359-2368, 2010 . caused upregulation of Cyt $b_{6} f$ iron-sulfur subunit, PsaD, and FNR and it could contribute to the improvement of the CEF-related protection for PSI and PSII. Moreover, the enhancement in the protein contents of $\mathrm{CAB}, \mathrm{OEE}$, and Cyt $b_{6} f$ iron-sulfur subunit in the trehalose-pretreated groups helped relieve the damage of heat stress to photochemical efficiency and the electron transfer rate. The upregulation of ATPase, Rubisco, and FBPA suggested that trehalose pretreatment could effectively ameliorate the heat stress-induced damage to the energy synthesis and carbon reaction processes.

Luo Y., Li F., Wang G.P. et al.: Exogenously-supplied trehalose protects thylakoid membranes of winter wheat from heatinduced damage. - Biol. Plantarum 54: 495-501, 2010.

Luo Y., Li W.M., Wang W.: Trehalose: Protector of antioxidant enzymes or reactive oxygen species scavenger under heat stress? - Environ. Exp. Bot. 63: 378-384, 2008.

Lyu J.I., Min S.R., Lee J.H. et al.: Overexpression of a trehalose6-phosphate synthase/phosphatase fusion gene enhances tolerance and photosynthesis during drought and salt stress without growth aberrations in tomato. - Plant Cell Tiss. Org. 112: 257-262, 2013.

Majoul T., Bancel E., Triboï E. et al.: Proteomic analysis of the effect of heat stress on hexaploid wheat grain: Characterization of heat-responsive proteins from non-prolamins fraction. Proteomics 4: 505-513, 2004.

Mamedov M.D., Petrova I.O., Yanykin D.V. et al.: Effect of trehalose on oxygen evolution and electron transfer in photosystem 2 complexes. - Biochemistry-Moscow + 80: 61-66, 2015.

Martin W., Herrmann R.G.: Gene transfer from organelles to the nucleus: how much, what happens, and why? - Plant Physiol. 118: 9-17, 1998.

Morgan-Kiss R., Ivanov A.G., Williams J. et al.: Differential thermal effects on the energy distribution between photosystem II and photosystem I in thylakoid membranes of a psychrophilic and a mesophilic alga. - BBA-Biomembranes 1561: 251265, 2002.

Pfündel E., Klughammer C., Schreiber U.: Monitoring the effects of reduced PS II antenna size on quantum yields of photosystems I and II using the Dual-PAM-100 measuring system. PAM Appl. Notes 1: 21-24, 2008.

Phee B.K., Cho J.H., Park S. et al.: Proteomic analysis of the response of Arabidopsis chloroplast proteins to high light stress. - Proteomics 4: 3560-3568, 2004.

Qiang X., Paulsen A.Q., Guikema J.A. et al.: Functional and ultrastructural injury to photosynthesis in wheat by high temperature during maturation. - Environ. Exp. Bot. 35: 43-54, 1995.

Richards A.B., Krakowka S., Dexter L.B. et al.: Trehalose: a review of properties, history of use and human tolerance, and results of multiple safety studies. - Food Chem. Toxicol. 40: 871-898, 2002.

Schreiber U.: Pulse-amplitude-modulation (PAM) fluorometry and saturation pulse method: an overview. - In: Papageorgiou G.C., Govindjee (ed.): Chlorophyll A Fluorescence. A Signature of Photosynthesis. Pp. 279-319. Springer, Dordrecht 2004. Silva J., Kim Y.J., Sukweenadhi J. et al.: Molecular charac- 
terization of 5-chlorophyll $a / b$-binding protein genes from Panax ginseng Meyer and their expression analysis during abiotic stresses. - Photosynthetica 54: 446-458, 2016.

Szymańska R., Dłużewska J., Slesak I. et al.: Ferredoxin:NADP+ oxidoreductase bound to cytochrome b6f complex is active in plastoquinone reduction: implications for cyclic electron transport. - Physiol. Plantarum 141: 289-298, 2011

Wahid A., Gelani S., Ashraf M. et al.: Heat tolerance in plants: An overview. - Environ. Exp. Bot. 61: 199-223, 2007.

Wang L., Liang W., Xing J. et al.: Dynamics of chloroplast proteome in salt-stressed mangrove Kandelia candel (L.) Druce. - J. Proteome Res. 12: 5124-5136, 2013.

Wang X., Cai J., Jiang D. et al.: Pre-anthesis high-temperature acclimation alleviates damage to the flag leaf caused by postanthesis heat stress in wheat. - J. Plant Physiol. 168: 585-593, 2011.
Wang Z.Y., Freire E., McCarty R.E.: Influence of nucleotide binding site occupancy on the thermal stability of the F1 portion of the chloroplast ATP synthase. - J. Biol. Chem. 268: 20785-20790, 1993.

Wingler A., Paul M.: The role of trehalose metabolism in chloroplast development and leaf senescence. - In: Biswal B., Krupinska K., Biswal U.C. (ed.): Plastid Development in Leaves during Growth and Senescence. Advances in Photosynthesis and Respiration. Pp. 551-565. Springer, New York 2013. Zhang H., Whitelegge J.P., Cramer W.A.: Ferredoxin:NADP+ oxidoreductase is a subunit of the chloroplast cytochrome b6f complex. - J. Biol. Chem. 276: 38159-38165, 2001.

Zhang L., Liu J.: Effects of heat stress on photosynthetic electron transport in a marine cyanobacterium Arthrospira sp. - J. Appl. Phycol. 28: 757-763, 2016. 